\title{
POLYNÔMES DE JACOBI, INTERPRÉTATION COMBINATOIRE ET FONCTION GÉNÉRATRICE
}

\author{
DOMINIQUE FOATA' ${ }^{1}$ ET PIERRE LEROUX ${ }^{2}$
}

\begin{abstract}
This classical generating function for the Jacobi polynomials is derived by purely combinatorial methods.
\end{abstract}

1. Introduction. Soit $\left(P_{n}^{(\alpha, \beta)}(x)\right)(n \geqslant 0)$ la suite des polynômes de Jacobi définis par

$$
(1-x)^{\alpha}(1+x)^{\beta} P_{n}^{(\alpha, \beta)}(x)=\left((-1)^{n} /\left(2^{n} n !\right)\right)(d / d x)^{n}\left[(1-x)^{n+\alpha}(1+x)^{n+\beta}\right] .
$$

Posant

$$
R=\left(1-2 x u+u^{2}\right)^{1 / 2},
$$

on sait depuis Jacobi que la fonction génératrice de ces polynômes est donnée par

$$
\sum P_{n}^{(\alpha, \beta)}(x) u^{n}=2^{\alpha+\beta} R^{-1}(1-u+R)^{-\alpha}(1+u+R)^{-\beta} \quad(n \geqslant 0) .
$$

On connait plusieurs démonstrations de la formule (1.3) ci-dessus (cf. Szegö [12, pp. 69-70], Rainville [9, §140], Carlitz [3]), toutes commentées avec beaucoup d'érudition par Askey [1], qui lui-même en propose une nouvelle en réactualisant une idée due à Hermite. Le but du présent article est d'abord de donner une interprétation combinatoire aux polynômes de Jacobi, ensuite de déduire directement de cette interprétation une démonstration combinatoire, donc complètement élémentaire, de l'identité (1.3).

Calculant la $n$-ème dérivée dans (1.1) par la règle de Leibniz, on obtient (cf. [12, p. 68])

$$
P_{n}^{(\alpha, \beta)}(x)=\sum\left(\begin{array}{c}
n+\alpha \\
n-j
\end{array}\right)\left(\begin{array}{c}
n+\beta \\
j
\end{array}\right)\left(\frac{x-1}{2}\right)^{j}\left(\frac{x+1}{2}\right)^{n-j} \quad(0 \leqslant j \leqslant n)
$$

Posant $(a)_{n}=1$ pour $n=0$ et $(a)_{n}=a(a+1) \cdots(a+n-1)$ pour $n \geqslant 1$, on peut définir les polynômes suivants, à coefficients entiers, dits homogènes de Jacobi

$$
\underset{n}{\mathrm{pp}_{n}(\alpha, \beta)}(X, Y)=\sum\left(\begin{array}{c}
n \\
i
\end{array}\right)(\alpha+1+j)_{i}(\beta+1+i)_{j} X^{i} Y^{i} \quad . .(i, j \geqslant 0, i+j=n) .
$$

Received by the editors February 11, 1982.

1980 Mathematics Subject Classification. Primary 33A65; Secondary 05A15.

Key words and phrases. Jacobi polynomials, generating functions, endofunctions, arborescences = rooted trees, Catalan numbers.

'Ce travail a été réalisé lorsque l'auteur était l'hôte de l'Université du Québec à Montréal.

${ }^{2}$ Avec l'appui financier du Ministère de l'éducation du Québec, subvention d'équipe EQ 1608. 
D’après (1.4) et (1.5), ils sont reliés aux polynômes de Jacobi par

$$
P_{n}^{(\alpha, \beta)}(x)=(1 / n !) \mathscr{P}_{n}^{(\alpha, \beta)}\left(\frac{x+1}{2}, \frac{x-1}{2}\right) .
$$

Définissant

$$
\circ R=\left(1-2(X+Y) u+(X-Y)^{2} u^{2}\right)^{1 / 2},
$$

nous établissons la formule suivante

$$
\begin{aligned}
& \sum \operatorname{vp}_{n}^{(\alpha, \beta)}(X, Y) u^{n} / n ! \\
& \quad=2^{\alpha+\beta} \mathcal{R}^{-1}(1-(X-Y) u+\Re)^{-\alpha}(1-(Y-X) u+\mathcal{R})^{-\beta} \quad(n \geqslant 0),
\end{aligned}
$$

d'où l'on tire (1.3) en posant $X=(x+1) / 2$ et $Y=(x-1) / 2$ et en utilisant (1.6). En fait, comme nous l'ont fait remarquer Richard Askey dans une communication privée et l'arbitre anonyme, les formules (1.3) et (1.8) sont équivalentes, puisque l'on a

$$
P_{n}^{(\alpha, \beta)}\left(\frac{X+Y}{X-Y}\right)(X-Y)^{n}=\mathscr{P}_{n}^{(\alpha, \beta)}(X, Y) / n !
$$

et qu'en remplaçant $u$ par $(X-Y) u$ et $x \operatorname{par}(X+Y) /(X-Y)$, on obtient (1.8) à partir de (1.3).

Les polynômes $\mathcal{P P}_{n}^{(\alpha, \beta)}(X, Y)$ apparaissent comme des polynômes générateurs de structures discrètes-ci-dessous appelées endofonctions de Jacobi-par un certain poids (cf. Théorème 2). La démonstration de (1.8), donc de (1.3), présentée dans les $\$ \S 3$ et 4 se fonde implicitement sur le fait que la série génératrice exponentielle de toutes les endofonctions de Jacobi est égale à l'exponentielle de la série génératrice exponentielle des endofonctions de Jacobi connexes. Cette démonstration s'appuie également sur les interprétations combinatoires des opérations courantes sur les séries génératrices comme la somme, le produit, la substitution, etc. (Voir, par exemple, $[2,5,11]$ et plus récemment $[7,8]$.) Elle a l'avantage de montrer pourquoi le second membre de (1.8) est un produit de trois séries (cf. formule (3.7)) et d'expliquer l'apparition de $\Re$ en (1.7) comme le radical d'un discriminant.

On note $|A|$ le cardinal d'un ensemble fini $A$ et $[n]$ l'intervalle $\{1,2, \ldots, n\}$ des entiers.

2. Endofonctions de Jacobi. Soient $S$ un ensemble fini et $f$ une endofonction de $S$, c'est-à-dire une application de $S$ dans $S$. On identifie habituellement $f$ avec son graphe associé, dont les sommets sont les éléments de $S$ et dans lequel un arc va de $s$ à $t$ si et seulement si $f(s)=t$. Comme il est bien connu (cf. [4, pp. 83-84; 5, pp. 102-107]) chaque composante connexe d'une endofonction contient un et un seul cycle.

DEFINITION 1. On appelle endofonction de Jacobi sur $S$ tout couple $\phi=((A, B), f)$ où

(i) $(A, B)$ est une partition ordonnée de $S$;

(ii) $f$ est une endofonction de $S$ dont les restrictions $\left.f\right|_{A}: A \rightarrow S$ et $\left.f\right|_{B}: B \rightarrow S$ aux parties $A$ et $B$ sont injectives. 
Soit $a(\phi)$ (resp. $b(\phi))$ le nombre de cycles de $f$ dont tous les sommets sont dans $A$ (resp. dans $B)$. Le poids de $\phi=((A, B), f)$ est défini par

$$
w(\phi)=(\alpha+1)^{a(\phi)}(\beta+1)^{b(\phi)} X^{|A|} Y^{|B|} .
$$

On note $\operatorname{Jac}(S)$ l'ensemble de toutes les endofonctions de Jacobi sur $S$.

THEOREME 2. Si $|S|=n$, on $a$

$$
\mathscr{P}_{n}^{(\alpha, \beta)}(X, Y)=\sum w(\phi) \quad(\phi \in \mathrm{Jac}(S)) .
$$

Démonstration. Etant donnée une partition $\left(A_{0}, B_{0}\right)$ de $S$ telle que $\left|A_{0}\right|=i$, $\left|B_{0}\right|=j$ et $i+j=n$ notons $w\left(A_{0}, B_{0}\right)$ le polynôme générateur, par $w$, des endofonctions de Jacobi $((A, B), f)$ sur $S$ telles que $A=A_{0}$ et $B=B_{0}$. D'après (1.5), il suffit de démontrer l'identité

$$
w\left(A_{0}, B_{0}\right)=(\alpha+1+j)_{i}(\beta+1+i)_{j} X^{i} Y^{j} .
$$

Or soient $g$ et $h$ deux injections de $A_{0}$ dans $S$ et de $B_{0}$ dans $S$, respectivement. Notons $c(g)$ et $c(h)$ leur nombre de cycles respectif et définissons leur poids par

$$
w(g)=(\alpha+1)^{c(g)} X^{i} \text { et } w(h)=(\beta+1)^{c(h)} Y^{j} .
$$

Il résulte de la Définition 1 que l'on a

$$
w\left(A_{0}, B_{0}\right)=\sum_{g} w(g) \cdot \sum_{h} w(h)
$$

où $g$ et $h$ décrivent, respectivement, l'ensemble des injections de $A_{0}$ dans $S$ et de $B_{0}$ dans $S$. Pour démontrer (2.3) il suffit donc d'établir le lemme suivant.

LEMME 3. Le polynôme générateur des injections $g$ de $A_{0}$ dans $S$ par $w$ (donné en (2.4)) est égal à $(\alpha+1+j)_{i} X^{i}$.

La démonstration du Lemme 3 est donnée dans [6]. Elle se fait facilement par récurrence sur $i$. Le cas $j=0$ est le comptage bien connu des permutations suivant le nombre de cycles [10, p. 71] et le cas $\alpha=0$ est le comptage direct des injections.

3. Séries génératrices. On définit le v-poids d'une endofonction de Jacobi $\phi=$ $((A, B), f)$ par

$$
v(\phi)=X^{|A|} Y^{|B|} .
$$

Le $v$-poids se déduit du $w$-poids en posant $\alpha=\beta=0$. On introduit alors les séries génératrices exponentielles des endofonctions de Jacobi selon le $v$-poids et selon le $w$-poids:

$$
\begin{array}{ll}
\Phi_{v}(u)=\sum\left(u^{n} / n !\right) \sum v(\phi) & (n \geqslant 0, \phi \in \mathrm{Jac}[n]), \\
\Phi_{w}(u)=\sum\left(u^{n} / n !\right) \sum w(\phi) & (n \geqslant 0, \phi \in \mathrm{Jac}[n]) .
\end{array}
$$

D'après le Théorème 2 on a l'identité

$$
\Phi_{w}(u)=\sum \mathscr{P}_{n}^{(\alpha, \beta)}(X, Y) u^{n} / n ! \quad(n \geqslant 0) .
$$

Nous allons montrer dans ce qui suit que $\Phi_{w}(u)$ est bien égal au second membre de (1.8). 
Si l'on représente une endofonction de Jacobi $\phi=((A, B), f)$ (comme dans la Figure 1, où pour plus de clarté on n'a pas indiqué les étiquettes des sommets) par un graphe orienté dont les arcs issus de sommets de $A$ (resp. de $B$ ) sont en trait continu (resp. pointillé), alors

(3.5) tout sommet est le but d'au plus un arc continu et d'au plus un arc pointillé.

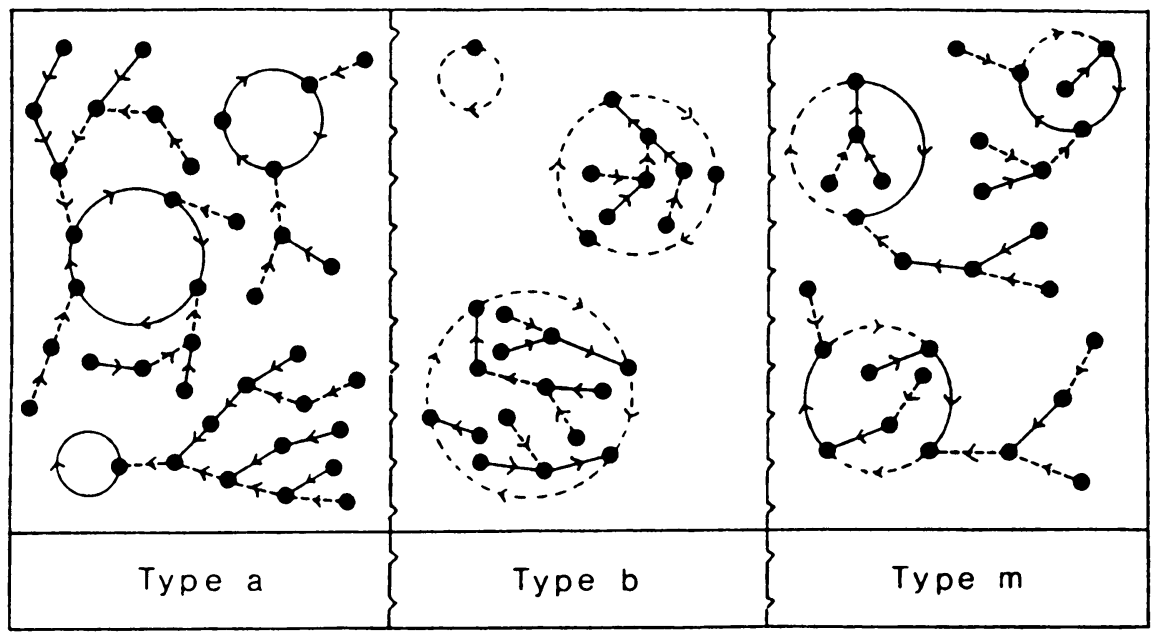

FIGURE 1

On distingue trois types d'endofonctions de Jacobi, dites de type a, de type b ou de type mixte, selon que tous les cycles qu'elles contiennent ont seulement des arcs continus, des arcs pointillés ou ont des arcs des deux sortes, respectivement; ceci détermine trois sous-ensembles de $\operatorname{Jac}(S)$, pour chaque ensemble fini $S$, ainsi que les séries génératrices exponentielles correspondantes: $\Phi_{a}(u), \Phi_{b}(u)$ et $\Phi_{m}(u)$, selon le $v$-poids, et $\Phi_{\alpha}(u), \Phi_{\beta}(u)$ et $\Phi_{\mu}(u)=\Phi_{m}(u)$ selon le $w$-poids.

LEMME 4. On a les identités

$$
\begin{aligned}
& \Phi_{v}(u)=\Phi_{a}(u) \Phi_{b}(u) \Phi_{m}(u), \\
& \Phi_{w}(u)=\Phi_{\alpha}(u) \Phi_{\beta}(u) \Phi_{m}(u)
\end{aligned}
$$

Demonstration. Comme le suggère la Figure 1, on peut partager le domaine $S$ d'une endofonction de Jacobi $\phi$ en trois parties, $S=S_{a}+S_{b}+S_{m}$, sur lesquelles la restriction de $\phi$ donne des endofonctions de Jacobi de type $\mathrm{a}, \mathrm{b}$ et $\mathrm{m}$ respectivement. Comme les $v$ - et $w$-poids se comportent multiplicativement par rapport à cette décomposition, le résultat découle de l'interprétation habituelle du produit de séries génératrices exponentielles (voir par exemple [7, Chapitre 6]).

LEMME 5. On a les identités

$$
\Phi_{\alpha}(u)=\left(\Phi_{a}(u)\right)^{\alpha+1}, \quad \Phi_{\beta}(u)=\left(\Phi_{b}(u)\right)^{\beta+1} .
$$


Demonstration. Si une endofonction de Jacobi $\phi$ de type a comporte $k=a(\phi)$ composantes connexes $\phi_{1}, \phi_{2}, \ldots, \phi_{k}$, on a

$$
w(\phi)=(\alpha+1)^{k} v\left(\phi_{1}\right) v\left(\phi_{2}\right) \cdots v\left(\phi_{k}\right) .
$$

Cette propriété multiplicative de $w$ entraine que

$$
\Phi_{\alpha}(u)=\exp \Phi_{\alpha, c}(u)=\exp \left((\alpha+1) \Phi_{a, c}(u)\right)=\left(\Phi_{a}(u)\right)^{\alpha+1},
$$

où $\Phi_{\alpha, c}(u)$ et $\Phi_{a, c}(u)$ sont les séries génératrices exponentielles des endofonctions de Jacobi connexes de type a selon le $w$ - et le $v$-poids, respectivement. La démonstration est la même pour les endofonctions de type $b$.

Pour démontrer (1.8) il suffit donc, en vertu des deux lemmes précédents, d'établir les identites

$$
\begin{gathered}
\Phi_{v}(u)=\mathscr{R}^{-1}, \\
\Phi_{a}(u)=2(1-(X-Y) u+G)^{-1}, \\
\Phi_{b}(u)=2\left(1-(Y-X) u+(R)^{-1} .\right.
\end{gathered}
$$

4. Arborescences de Jacobi. Soit $C_{a}(u)$ (resp. $C_{b}(u)$ ) la série génératrice exponentielle selon le $v$-poids des contractions de type a (resp. de type b), c'est-à-dire des endofonctions de Jacobi de type a (resp. de type b) connexes, dont le cycle est de longueur 1 .

LEMME 6. On a les identités

$$
\begin{gathered}
\Phi_{a}(u)=\left(1-C_{a}(u)\right)^{-1}, \\
\Phi_{b}(u)=\left(1-C_{b}(u)\right)^{-1}, \\
\Phi_{v}(u)=\left(1-\left(C_{a}(u)+C_{b}(u)\right)\right)^{-1} .
\end{gathered}
$$

Demonstration. L'identité (4.1) exprime le fait qu'une endofonction de Jacobi de type a est une permutation (au sens fonctionnel) de contractions de type a (cf. [5, pp. 110-111 ou 7, Figure 12] pour une illustration de ce principe dans un cas connexe). Les identités (4.2) et (4.3) sont semblables, une contraction étant soit de type a, soit de type $b$.

Il reste à calculer effectivement $C_{a}(u)$ et $C_{b}(u)$. C'est une légère extension du calcul traditionnel des nombres de Catalan [4, p. 65] qui va nous le donner. Appelons arborescence de Jacobi toute arborescence non étiquetée $\psi$ dont les arcs, tous orientés vers la racine, sont soit "continus", soit "pointillés", et qui satisfait à la condition (3.5) (cf. Figure 2). Désignons par $c(\psi)$ et $p(\psi)$ le nombre d'arcs continus et pointillés de $\psi$, respectivement, et définissons le $v$-poids de $\psi$ par

$$
v(\psi)=X^{c(\psi)} Y^{p(\psi)} \text {. }
$$

Enfin, notons $A(u)$ la série génératrice ordinaire des arborescences de Jacobi selon le $v$-poids. Il est facile de voir que

$$
C_{a}(u)=X u(1+Y A(u)), \quad C_{b}(u)=Y u(1+X A(u)) .
$$

La série $A(u)$ satisfait, elle, à l'identité donnée dans le lemme suivant. 
LEMME 7. On $a$

$$
A(u)=u(1+X A(u))(1+Y A(u))
$$

Demonstration. La relation (4.6) exprime le fait qu'une arborescence de Jacobi est constituée d'une racine à laquelle sont attachées deux branches, chacune étant soit vide, soit constituée d'une arborescence de Jacobi rattachée à la racine par un arc continu dans un cas et pointillé dans l'autre.

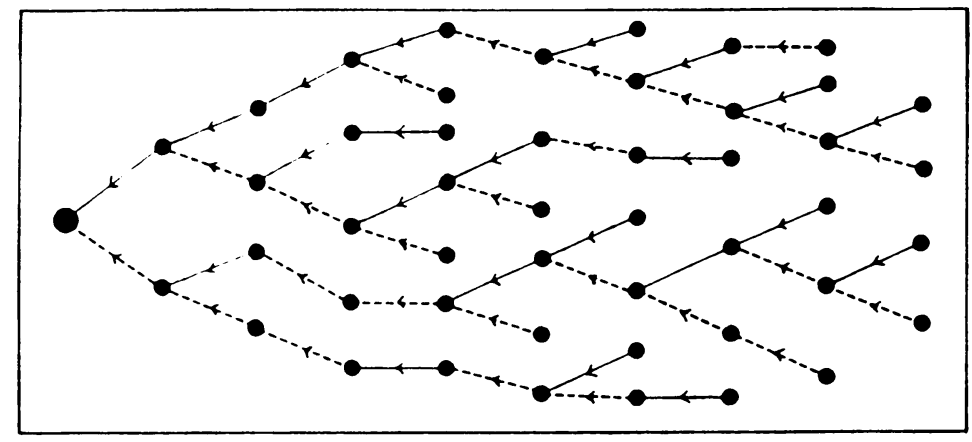

FIGURE 2

Le calcul qui suit est calqué sur celui de la série génératrice des nombres de Catalan et s'y réduit pour $X=Y=1$. De (4.6) on déduit

$$
X Y u(A(u))^{2}+((X+Y) u-1) A(u)+u=0 .
$$

Le radical du discriminant de cette équation quadradique en $A(u)$ est, après calcul, donné précisément par (1.7) et on en conclut, prenant la solution qui s'annule en $u=0$, que

$$
A(u)=(1-(X+Y) u-\text { o }) / 2 X Y u .
$$

Pour compléter la démonstration de (3.11), (3.12) et (3.13) et donc de (1.8), il suffit maintenant de substituer (4.8) dans (4.5) pour obtenir une forme close pour $C_{a}(u)$ et $C_{b}(u)$, puis de substituer ces expressions dans (4.3), (4.1) et (4.2).

REmarQue. La condition (3.5) affirme que la fibre de tout sommet est munie d'une structure binaire et planaire, de série génératrice

$$
S(u)=1+X u+Y u+X Y u^{2},
$$

compte-tenu du $v$-poids. Les endofonctions et arborescences de Jacobi peuvent donc être considérées comme des endofonctions et arborescences $S$-enrichies, dans la terminologie de G. Labelle [8, 7, §2.4].

\section{REFERENCES}

I. R. Askey, Jacobi's generating function for Jacobi polynomials, Proc. Amer. Math. Soc. 71 (1978). $243-246$.

2. E. A. Bender and J. R. Goldman, Enumerative uses of generäting functions, Indiana Univ. Math. J. 20 (1971), 753-765.

3. L. Carlitz, The generating function for the Jacobi polynomials, Rend. Sem. Mat. Univ. Padova 38 (1967), 86-88. 
4. L. Comtet, Analyse combinatoire, Tome I, Presses Universitaires de France, Paris, 1970; English ed., Advanced combinatorics, Reidel, Dordrecht and Boston, 1974.

5. D. Foata, La série génératrice exponentielle dans les problèmes d'énumération, Presses de l'Universitè de Montréal, Montréal, 1974.

6. D. Foata and V. Strehl, Combinatorics of Laguerre polynomials, prépublication, Univ. Strasbourg, 1981.

7. A. Joyal, Une théorie combinatoire des séries formelles, Adv. in Math. 42 (1981), 1-82.

8. G. Labelle, Une nowvelle démonstration combinatoire des formules d'inversion de Lagrange, Adv. in Math. 42 (1981), 217-247.

9. E. D. Rainville, Special functions, Chelsea, New York, 1960.

10. J. Riordan, An introduction to combinatorial analysis, Wiley, New York, 1958.

11. R. P. Stanley, Generating functions, Studies in Combinatorics (G. C. Rota, ed.), Math. Assoc. Amer. Studies in Math., vol. 17, 1978, pp. 100-141.

12. G. Szegö, Orthogonal polynomials, Amer. Math. Soc. Colloq. Publ., vol. 23, Amer. Math. Soc., Providence, R. I., 1939 (second printing of the fourth edition: 1978).

Departement de Mathematique, Universite de Strasbourg, 7 Rue Rene Descartes, F 67084 STRASBOURG, France

Departement de Mathematiques, Universite du Quebec a Montreal, Case Postale 8888 , Succursale “A”, Montreal, Quebec, Canada H3C 3P8 